\title{
Lost in uncertainty? Sozial entscheiden und organisieren in der Sozialwirtschaft
}

\author{
Herbert Effinger ${ }^{1}$ \\ Angenommen: 5. Oktober 2021 / Online publiziert: 19. November 2021 \\ (c) Der/die Autor(en) 2021
}

\section{Zusammenfassung}

Dieser Beitrag der Zeitschrift „Gruppe. Interaktion. Organisation. (GIO)“ behandelt den Wohlfahrtsmix von Gemeinschaft, Sozialstaat und Markt mit ihren widersprüchlichen Regulationsprinzipien in der Sozialwirtschaft. Im Zusammenspiel solidarischer, sozialstaatlicher und ökonomischer Regulation werden Situationen von Ungewissheit, Ambiguität, Unsicherheit und Ambivalenz sowie wenig geeignete Bewältigungsstrategien, wie ein defensives Vermeidungsverhalten und dysfunktionale Organisationskulturen, begünstigt. In dem Kontext stehen die Organisationen und ihre Sorgearbeitenden vor der Herausforderung Komplexität und damit verbundener Ungewissheit und Unsicherheit bewältigen zu müssen und sich dabei vor unproduktiven Komplexitätsreduktionen, etwa in Form von moralisierender Vereindeutigungen und Simplifizierungen, zu schützen. Es gilt die widersprüchlichen Dynamiken dieses Handlungsfeldes proaktiv zu auszubalancieren und zu bewältigen.

Schlüsselwörter Ungewissheit · Unsicherheit · Sozialwirtschaft · Wohlfahrtsmix · Soziale personenbezogene Dienstleistungen · Soziale Arbeit · Beratung · Sozialmanagement · Defensives Vermeidungsverhalten · Wertekonflikte ·

Selbstkompetenz

\section{Deciding and organizing socially in the social economy}

\section{Abstract}

This article in the magazine "Gruppe. Interaktion. Organization. (GIO)" deals with the welfare mix of community, welfare state and market with their contradicting principles of regulation in the social economy. In the context of the interrelation between solidarity, welfare state and economic regulation, the actors often favor situations of uncertainty, ambiguity, insecurity and ambivalence as well less suitable coping strategies, such as defensive avoidance behavior and dysfunctional organizational cultures. In this context, the organizations and care workers are confronted with the challenge of overcoming complexity and associated uncertainty and to avoid unproductive complexity reductions, like moralizing conflation and simplification. It is important to cope and balance adequately with the contradictory dynamics of this field of action.

Keywords Uncertainty $\cdot$ Insecurity $\cdot$ Social economy $\cdot$ Welfare mix $\cdot$ Social personal services $\cdot$ Social work . Counseling · Social management · Defensive avoidance behavior · Value conflicts uncertainty · Social economy · Welfare mix $\cdot$ Social work $\cdot$ Counselling $\cdot$ Social management $\cdot$ Support management $\cdot$ Conflict of values $\cdot$ Self-competence

Prof. (em) Dr. Herbert Effinger

effinger.h@t-online.de

1 Dresden, Deutschland 


\section{Einleitung}

Die Sozialwirtschaft ${ }^{1}$ gilt als einer der expansivsten Arbeitsmärkte (Heinze 2011; Grunwald und Langer 2018; Kolhoff und Grunwald 2018; Schneiders 2020, S. 25 ff.; Wöhrle et al. 2019). ${ }^{2}$ Dieser Wirtschaftsbereich lässt sich als ein sektorenübergreifendes Funktionssystem sozialstaatlicher Daseinsvorsorge und Daseinsfürsorge beschreiben. Darin ist das ,individuelle und gemeinsame Wohlergehen von Menschen“ das primäre Ziel. Dazu zählen alle „personen- und gemeinschaftsbezogenen sozialen Dienste“, die von öffentlichen Trägern, freien Wohlfahrtsverbänden und Vereinen, Kirchen und kommerziellen Unternehmen, ,nicht primär erwerbs- und gewinnorientiert, sondern in Deckung eines humanen Bedarfs" betrieben werden (Wendt 2017, S. 856f.). Die Lebenslagen der als bedürftig angesehenen Bürger, benachteiligten Gruppen und Gemeinwesen sind durch materielle und psychosoziale Unsicherheit geprägt. Zur Bearbeitung dieser Problemlagen, sind die Sorgearbeitenden auf eine aktive Mitwirkung (Koproduktion) ihrer Nutzer und Nutzerinnen angewiesen (Effinger 1993).

Es handelt sich um ein hybrides Funktionssystem (siehe Abb. 1), in dem Regulationsmechanismen aus Gemeinschaft, Sozialstaat und Markt miteinander verbunden sind (Evers und Ewert 2010). An die Sorgearbeitenden stellt das besondere Kompetenzanforderungen (Heiner 2007, 2010; Müller 2012; Zobrist und Kähler 2017). ${ }^{3}$

Etwa $80 \%$ der Beschäftigten in der Sozialwirtschaft sind Frauen. Überdurchschnittlich viele der Beschäftigten arbeiten in Teilzeit und in prekär befristeten Beschäftigungsverhältnissen (Schneiders 2020). Die Erwerbseinkommen sind deutlich geringer als in anderen Wirtschaftsbereichen

\footnotetext{
${ }^{1}$ Ich grenze hier Sozialwirtschaft auf jene Bereiche ein, in denen im Rahmen sozialpolitischer Rahmenbedingungen primär sozialarbeiterische und sozialpädagogische Dienste angeboten werden. Erzieherische und unterrichtende Aufgaben wie im Bildungsbereich und pflegerische Aufgaben wie im Gesundheitsbereich gehören hier nicht zum dominanten Handlungsmodell (Effinger 2018, S. 17 ff., 2021a, S. $117 \mathrm{ff}$.) Alle Mitarbeitenden in diesem Funktionssystem, die soziale personenbezogene Dienstleistungen anbieten, bezeichne ich mit dem Oberbegriff als Sorgearbeitende. Sorgearbeitende mit einem akademischen Abschluss, i.d.R. als Sozialpädagoge, Sozialpädagogin, Sozialarbeiter oder Sozialarbeiterin, nenne ich Sozialarbeitende.

2 Über die Anzahl der in der deutschen Sozialwirtschaft vorhandenen sozialversicherungspflichtigen Beschäftigungsverhältnisse gibt es aufgrund mangelnder Statistiken keine präzisen Daten. Wenn man den Bereich auf „Expert*innen im Berufsfeld Soziale Arbeit“ (Mazari 2021, S. 186) begrenzt, kann man von bis zu 300.000 Beschäftigen ausgehen. Davon haben nach Mazari gut Zweidrittel einen akademischen Berufsabschluss. Fasst man den Bereich weiter, kommen Schätzungen auf 2,5 bis 3 Mio. Beschäftigte mit einem Akademikeranteil von 10-20\%.

${ }^{3}$ Im Sozialrecht (SGB) sind die Beziehungen in einem sozialrechtlichen Dreiecksverhältnis von Leistungsträgern (Institutionen des Sozialstaates), Leistungserbringern (Anbieter von sozialen Dienstleistungen in der Sozialwirtschaft) und den Leistungsberechtigten (Adressaten und Adressatinnen) geregelt.
}

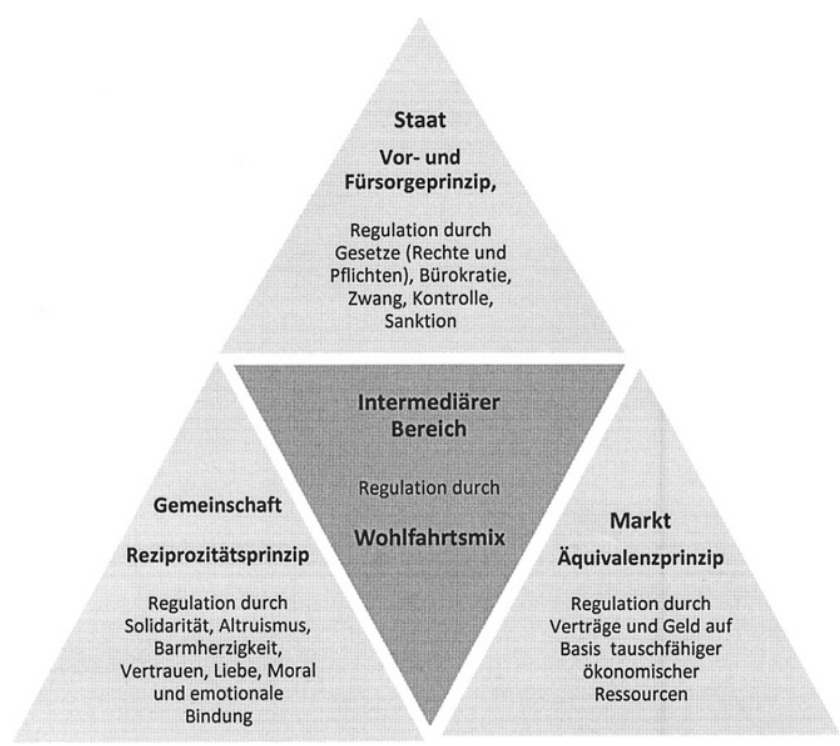

Abb. 1 Idealtypische Regulationsbereiche und -prinzipien in der Sozialwirtschaft. (C) Herbert Effinger (Effinger 2021a, S. 135; Effinger 2018, S. 21f.))

(Mazari 2021). Komplexe und wenig durschaubare informelle Strukturen sowie prekäre Existenzformen der Träger prägen oft dieses Handlungsfeld (Wöhrle et al. 2019). Die Arbeitsabläufe sind meist nur gering standardisiert, und Routineabläufe bilden eher die Ausnahme. Wechselseitiges Vertrauen und gute Beziehungen in dieser koproduktiven Wohlfahrtsproduktion (Effinger 2021a, S. $147 \mathrm{ff}$.) gelten als wesentliche Grundlagen für deren Wirksamkeit und Gelingen (Böhle 2017; Gahleitner 2017; Schröder 2017; Wagenblass 2018). Dem konstruktiven Umgang mit eigenen und fremden Emotionen und Affekten und daraus resultieren Ambivalenzen und sozialen Konflikten kommt eine herausragende Bedeutung zu.

Von den Sorgearbeitenden wird erwartet, dass sie im hohen Maße Experten bzw. Expertinnen für Ungewissheitsbewältigung sind. Obwohl die objektiven, lebenslagenbedingten Unsicherheiten der Adressaten und Adressatinnen außerhalb der Kompetenz- und Zuständigkeitsbereiche der Sorgearbeitenden liegen, sollen letztere dabei helfen, die Lebensrisiken ihrer Adressaten und Adressatinnen zu minimieren und deren Lebensführungskompetenz zu optimieren. Unsicherheit ist nicht nur ein dieses Handlungsfeld objektiv prägendes Phänomen, sondern auch ein subjektives Gefühl, dass in einer von Ungewissheit geprägten Situation entstehen kann aber nicht entstehen muss (Effinger 2021a, S. 10 ff.) Ich unterscheide hier grundsätzlich zwei Formen von Ungewissheit, die mit Unsicherheit verbunden sein können:

1. Personale Ungewissheit und Unsicherheit in psychischen Systemen über die Einschätzung von Personen und Situa- 
tionen und über den Ausgang von sich daraus ergebenden Interaktionen und Interventionen.

2. Organisationale Ungewissheit und Unsicherheit über die Stärke, Dynamik und Richtung divergierender Kräfte in sozialen Systemen.

Diese Formen von Ungewissheit und Unsicherheit gehören in der Arbeit mit den Adressaten und Adressatinnen, innerhalb der eigenen Organisation sowie zwischen den beteiligten Organisationen im Hilfesystem zur charakteristischen Struktur der Arbeit in diesem hybriden Funktionssystem (Kurz-Adam 2018). Jedoch wird diese Problematik in den meisten Handbüchern für das Sozialmanagement vernachlässigt. Oftmals werden rein rationalistisch ausgerichtete, betriebswirtschaftliche Konzepte ohne Anpassung an die strukturellen und emotionalen Besonderheiten dieses Wirtschaftsbereiches übernommen (Evers et al. 2011; Hackenberg und Empter 2011; Brinkmann 2014; Gesmann und Merchel 2019). Auch in der Organisationssoziologie spielt diese Thematik nur eine marginale Rolle (Apelt und Senge 2015).

Nach Harrison und Lomi (2015). sind die klassischen Managementmodelle nicht geeignet, Entscheidungsverhalten in komplexen und von Ungewissheit und Unsicherheit geprägten Systemen hinreichend zu erfassen und in eine zielführende Richtung zu beeinflussen. Ihre Problemanalysen sind oft $\mathrm{zu}$ eindimensional und nicht in der Lage, mit multiplen Zielstellungen umzugehen. Ausgehend von rein rationalistischen Entscheidungsmodellen blenden diese Managementkonzepte die Widersprüche dieses Handlungsfeldes weitgehend aus und begünstigen so eine unzureichende Entscheidungskultur in den Organisationen.

Vor diesem Hintergrund gehe ich der Frage nach, vor welchen Herausforderungen Organisationen der Sozialwirtschaft mit ihren spezifischen Ungewissheits- und Unsicherheitspotentialen stehen. In welchem Maße unterstützen oder behindert der Wohlfahrtsmix Sorgearbeitende darin, ihren gesellschaftlichen Auftrag zu erfüllen? Können die Sorgearbeitenden und ihre Leitungs- und Führungskräfte mit den widersprüchlichen Systemlogiken und -dynamiken im Sinne ihres gesellschaftlichen und professionellen Auftrags kreativ umgehen oder verlieren sie sich eher in defensiven Vermeidungsstrategien?

Die hier aufgeworfenen Beobachtungen und Thesen beruhen auf empirischen Daten und theoretischen Analysen über die strukturellen Bedingungen und Verhältnisse in diesem Bereich sowie auf meinen Erfahrungen als Sozialarbeiter, Supervisor, Organisationsberater und Coach mit Sorgearbeitenden und häufig anzutreffenden Verhaltensweisen im Umgang mit diesen Verhältnissen. ${ }^{4}$

\footnotetext{
${ }_{4}^{4}$ Wenn in diesem Text eher „Negativbeispiele“ erwähnt werden, soll das nicht heißen, dass es auch positive Beispiele gibt.
}

Ich werde zunächst die widersprüchlich wirkenden Regulationsprinzipien der Sozialwirtschaft darstellen und dann auf den Charakter der in diesem Bereich typischen Hilfebeziehungen und deren Wirkungen und Nebenwirkungen für das Entscheiden und Handeln der Sorgearbeitenden eingehen. Abschließend ziehe ich ein paar Schlussfolgerungen für die Forschung, Aus- Fort- und Weiterbildung und die Praxis.

\section{Sozialwirtschaft zwischen egalitärer und elitärer Ordnungs- und Sozialpolitik und widersprüchlichen Regulierungsprinzipien ${ }^{5}$}

Bis vor einigen Jahren galten Soziales und Wirtschaft im Kontext sozialer personenbezogener Dienstleistungen noch als Gegensatz. Deren Anbieter verstanden sich als Organisationen, welche die negativen Folgen marktwirtschaftlicher Regulation und kapitalistischer Produktionsweisen ausgleichen oder abmildern. Markt- und Betriebswirtschaft galten als Teil des Problems und nicht als Teil der Lösung. Je nach Interessenslage, Werteorientierung und ordnungspolitischer Perspektive verbinden sich mit diesem hybriden Funktionssystem sehr unterschiedliche Interessen, Hoffnungen oder Befürchtungen. Aus meiner Sicht geht es hier um ein Spannungsfeld zwischen egalitären und elitären Strategien der Wohlfahrtsproduktion, um Strategien, die entweder auf strikt universalen oder wertrationalen Prinzipien von Gerechtigkeit und Gleichheit oder aber auf partikularen und zweckrationalen Prinzipien der Bevorzugung ausgewählter (elitärer) Gruppierungen beruhen. Diese Ausrichtungen finden sich auch, in unterschiedlicher Gewichtung, bei den verschiedenen Akteuren und Akteurinnen innerhalb dieses Funktionssystems wieder. ${ }^{6}$

Neokonservative und neoliberale Protagonisten vertreten m.E. einen proprietären Elitarismus. im Rahmen gesellschaftlicher Wertschöpfung stehen aus ihrer Sicht stehen den „Fleißigen“ und den Besitzenden als den „Leistungsträgern" mehr Privilegien zu und verdienen einen größeren Benefit als andere, die von ihnen als weniger leistungsstark und produktiv betrachtet werden. In der Tradition sozialdemokratischer Konzepte liegt der Fokus dagegen auf der Herstellung von Chancengleichheit und Gerechtigkeit in einem strukturell von Ungleichheit und Ungerechtigkeit verursachenden Wirtschaftssystem. Diese Strategie bezeichne ich

\footnotetext{
5 Die Kategorien ,egalitäre und elitäre Ordnungs- und Sozialpolitik“ verwende ich, um gegensätzliche Grundausrichtungen dieser Politik zwischen eher auf universalistischen oder eher auf partikularistischen beruhenden Prinzipien und Orientierungen zu kennzeichnen.

${ }^{6}$ Zur Diskussion um die Entwicklung der Sozialwirtschaft siehe: Kolhoff und Grunwald (2018); Grunwald und Langer (2018).
} 
als einen kompensatorischen-Egalitarismus, der darauf abzielt, mit den Mittel der Umverteilung soziale Ungleichheit zu vermeiden bzw. auszugleichen. Von einem kooperativen Egalitarismus spreche ich bei basisdemokratischen und genossenschaftlichen Konzepten. Sie streben die Minderung oder Aufhebung des Interessengegensatzes von Produzenten und Konsumenten durch mehr Selbstbestimmung sowie mehr Teilhabe und Teilnahme der Mitarbeitenden und Nutzer und Nutzerinnen an (Effinger 1990).

In den professionstheoretischen Diskursen um „Soziale Arbeit als Dienstleistung“ (Oechler 2009) werden die Chancen und Grenzen anwaltlicher Ansätze und professioneller Autonomie zwischen Markt und Ökonomie ausgeleuchtet. Auf der Grundlage universell gültiger Menschenrechte sollen die Adressaten und Adressatinnen rechtlich und ökonomisch in die Lage versetzt werden, ein Wunsch- und Wahlrecht bei der Auswahl von für sie geeigneten Angeboten auszuüben. Dabei handelt es sich m.E. um einen advokarischen Egalitarismus. Neben den Nutzerrechten sollen auch die Rechte der Sozialarbeitenden in Form eigener Professionsdomänen und exklusiver Zuständigkeit ausgeweitet werden (Effinger und Körber 1994; Schütze 2021). Bei den Religionsgemeinschaften und den traditionellen Wohlfahrtsverbänden gibt es aus meiner Sicht sowohl Formen eines moralischen Elitarismus (Angebote nur für Gemeinschaftsmitglieder) als auch eines am Gemeinwohl orientierten Egalitarismus (Angebote für alle Bürger).

\subsection{Die neoliberale Wende - oder die Verbetriebswirtschaftlichung und Vermarktlichung des Sozialen}

Etwa seit Beginn der 80ziger Jahre wendete sich die Politik in den westlichen Industrieländern immer stärker einer neoliberalen und neokonservativen Ordnungspolitik zu (Aldred 2020). Marktwirtschaftliche und betriebswirtschaftliche Konzepte und Instrumente sollten die Defizite gemeinschaftlicher und öffentlicher Daseinsvor und -fürsorge beseitigen. Die Nutzer und Nutzerinnen sozialstaatlicher Leistungen, inkl. ihrer Partner und Angehörigen, sollen stärker in die Verantwortung zur Bewältigung ihrer Lebensrisiken genommen werden. Während sich öffentliche Träger immer mehr als Anbieter sozialer personenbezogener Dienstleistungen zurückzogen, entstanden seit Beginn der siebziger Jahre des 20. Jh. neben den traditionellen Wohlfahrtsverbänden viele neue freigemeinnützige Vereine und Selbsthilfegruppen, die dann vielfach unter das Dach des pluralistisch ausgerichteten Paritätischen Wohlfahrtsverbandes (DPWV) geschlüpft sind. Mit diesen ,alternativen“ Vereinen verbanden sich anfänglich zivilgesellschaftliche Hoffnungen auf eine Demokratisierung der Wohlfahrtsproduktion durch eine Bürgergesellschaft von unten (Bode et al. 2008).
Seit Beginn der neunziger Jahre des $20 \mathrm{Jh}$. verloren die traditionellen privilegierten Wohlfahrtsverbänden quasi ihr durch das Subsidiaritätsprinzip begründete Oligopol als Leistungserbringer Sozialer Dienste. Das Handlungsfeld wurde nun auch für privatkommerzielle Anbieter zugänglich. Mit zunehmender Konkurrenz entwickelten sich die Wohlfahrtsverbände und ihre Organisationseinheiten im Rahmen der sogenannten „Neuen Steuerung“ immer mehr zu Sozialunternehmen - die konfessionellen ebenso wie jene die aus der Tradition der Arbeiterbewegung stammen und auch einige Mitgliedsorganisationen des DPWV. So wurden immer mehr Sorgearbeitende zu „Social Entrepreneurs“ (Brinkmann 2014; Hackenberg und Empter 2011). Aus einem vormals reziproken „Liebesdienst“ im Rahmen von Gemeinschaft (Familie, Nachbarschaft, kleine Wertegemeinschaften) entstand ein Wohlfahrtsmix, dessen Angebote immer öfter zur Ware wurden und von Verdinglichungs- und Objektivierungsprozessen des Marktes, direkt oder indirekt, mitgeprägt werden. Kommunen und andere Öffentliche Träger als unmittelbare Leistungserbringer Sozialer Dienste zogen sich immer mehr zurück.

\subsection{Transformation der Leitbilder des Gemeinwohls in Instrumente des Sozialmarketing}

Die traditionellen Freien Träger (Wohlfahrtsverbände) begannen nun, sich diesen veränderten Rahmenbedingen anzupassen, implementierten Qualitätssicherungsprogramme und modernisierten ihre Leitbilder, um sich auf den neuen Sozialmärkten zu behaupten. Hintergrund dieser Bemühungen war auch die Auflösung ihrer traditionellen Milieus und ein damit einhergehender Bedeutungsverlust ihrer traditionellen Wertesysteme. Je weniger das Profil der jeweiligen Wertegemeinschaft in der pluralisierten Gesellschaft noch erkennbar war und entsprechende Bindekräfte entfaltete, desto mehr ließ die Bedeutung dieser Wertesysteme für die Arbeitsplatzwahl der Beschäftigten und für die Angebotsauswahl ihrer Adressaten nach. Aus gemeinwohlorientierten Leitbildern konfessionell und sozialdemokratisch geprägter Gemeinschaften wurden nun Instrumente des Sozialmarketings.

Im Zuge dieses Shifts von der Fürsorge- zu einer Dienstleistungsorientierung gab es durchaus Professionalisierungsfortschritte und Qualitätsverbesserungen. Man begann, sich von obrigkeitsstaatlichen Eingriffen abzukehren und ein Mehr an Partizipation und Selbstbestimmung der Adressaten und Adressatinnen zu ermöglichen. Es gelang aber nicht, die immer weiter steigenden Sozialstaatskosten zu senken. Ebenso fielen die Autonomiegewinne der Sozialarbeitenden gegenüber juristisch medizinisch, psychologisch und ökonomisch qualifizierten Professionellen insgesamt eher bescheiden aus. Kam den Leitbilddebatten anfangs noch eine innerorganisatorische Klärungsfunktion 
zu, bei denen verkrustete und nicht mehr zeitgemäße Organisations- und Angebotsstrukturen in Frage gestellt und verändert wurden, so spielten diese renovierten Leitbilder in den folgenden Jahren eine immer geringere Rolle.

\subsection{Verschiebung der Kriterien von sozialer zu ökonomischer Effizienz und Effektivität}

Mit der Einführung markt- und betriebswirtschaftlichen Instrumente wurden die Kriterien von Effizienz und Effektivität immer mehr in Richtung ökonomischer Kriterien verschoben. Auf der unmittelbaren Handlungsebene in den Organisationen werden diese jedoch von den Sorgearbeitenden überwiegend als hinderlich für das Gelingen des eigenen Auftrages eingeschätzt. Nach meinen Erfahrungen verstärkte sich der Widerspruch zwischen öffentlich proklamierten und von den Leitungen vertretenen Positionen einerseits und dem faktischen Handeln der Sorgearbeitenden anderseits.

\subsection{Ein Fallbeispiel ${ }^{7}$}

Das schwierige Zusammenspiel der unterschiedlichen Regulationsperspektiven illustriert das folgende Fallbeispiel aus einer Teamsupervision in einer Werkstadt für Menschen mit Behinderung (WfbM) des Diakonischen Werkes (DW). Der Betrieb wird in der Rechtsform einer gemeinnützigen GmbH geführt:

In der Werkstatt werden von den Beschäftigten (Menschen mit Behinderung) im Auftrag eines Automobilkonzerns Teile für die PKW Produktion gefertigt. Diese Arbeit ist Teil eines Rehabilitationsauftrages zur Integration in den Arbeitsmarkt und zur gesellschaftlichen Teilhabe nach dem Bundesteilhabegesetz (BTHG).

Aus Gründen des Verbots von Wettbewerbsverzerrungen durch öffentliche Subventionen entspricht der Produktionspreis dieser Teile in etwa dem Marktpreis, den auch ein kommerzieller Anbieter erwarten könnte. Für den Autokonzern ist ein solches Geschäft attraktiv, weil er diesen Auftrag zu 50\% auf seine Abgaben zur Schwerbehindertenquote anrechnen lassen kann. Die realen Produktionskosten in der WfbM liegen in aller Regel über den Marktpreisen, da die Werkstätten und ihre Klientel über eine vergleichsweise geringere Produktivität verfügen. Für den Fall, dass die Teile nicht in der vereinbarten Qualität, Menge und zu den vereinbarten Zeiten geliefert werden, werden Vertragsstrafen fällig. In den Stückpreis gehen nicht

\footnotetext{
7 Diese Fallvignette beruht Erfahrungen in verschiedenen Beratungsprozessen dieses Handlungsfeldes und ist in dieser Form konstruiert.
}

alle tatsächlichen Produktionskosten ein. Die Einrichtung der Arbeitsplätze wird von der Bundesanstalt für Arbeit (Ausbildung) und aus dem Haushalt des Bundesministeriums für Arbeit und Soziales (Beschäftigung) finanziert. Dazu gehört auch die Einrichtung eines Sozialdienstes. Die Werkstatt muss $70 \%$ der Einnahmen aus dem Teileverkauf an die Beschäftigten auszahlen. Der Rest kann für Investitionen genutzt werden. Die Beschäftigen sind keine sozialversicherungspflichtigen Arbeitnehmer und haben nur arbeitnehmerähnliche Rechte. Sie erhalten ein monatliches Entgelt zwischen 200 und $300 €$. Da dies bei weitem nicht ausreicht, um deren Lebensunterhalt zu bestreiten, bekommen sie zusätzlich Transferzahlungen vom Sozialamt (SGB IX). ${ }^{8}$

Angebote in der Werkstatt, die über das gesetzliche Maß hinausgehen, wie beispielsweise besondere sozial- und freizeitpädagogische und nicht unmittelbar produktionsbezogene Angebote, werden durch Zuwendungen des DW, aus privaten Spenden und von gemeinnütziger Stiftungen sowie Abgaben bei Glücksspielen finanziert. Neben den Fachanleitern in der Produktion ist der Sozialdienst mit Sozialarbeitenden für die sozialen Belange ihrer Beschäftigten und auch für die Bearbeitung von Konflikten im Betrieb und zwischen den Mitarbeitern und Mitarbeiterinnen zuständig. Daneben kümmern sich vereinzelt Ehrenamtliche um einzelne Klienten. In diesem Fall ist eine Ehrenamtliche beteiligt, die der örtlichen Kirchgemeinde angehört und sich in der Freizeit darum bemüht, Begegnungen zwischen Gemeindemitgliedern und Klienten zu ermöglichen.

Der Beschäftigte A., ein Mann mit einer psychischen Behinderung, kann als einziger der Beschäftigten eine für die Produktion der Autoteile erforderliche Maschine bedienen. Im familiären Umfeld des A. sind nun kürzlich Konflikte aufgetreten und haben seine Präsenz in der Werkstatt und seine Arbeitsleistung stark beeinträchtigt. Nachdem es zu Produktionsausfällen gekommen ist und die Werkstatt ihre Liefertermine nicht einhalten konnte, übt der Geschäftsführer, ein Betriebswirt, auf die Fachanleiter und diese auf A. erheblichen Druck aus. Gleichzeitig drängt der Geschäftsführer die zuständige Sozialarbeiterin $\mathrm{H}$. Sie soll dafür sorgen, dass A. wieder fit für die Arbeit wird, da sonst erhebliche Vertragsstrafen des Automobilkonzerns drohen. Der zuständige Fachanleiter, der die Maschine im Bedarfsfall auch bedienen könnte, ist seit ein paar Tagen im Jahresurlaub. Nachdem der Druck auf A. immer größer wurde, hat A. sich für mehre Wochen krankschreiben lassen. Der Automo-

\footnotetext{
${ }^{8}$ Näheres siehe BAG WfbM (2021).
} 
bilkonzern macht nun die Vertragsstrafe geltend und droht damit, der WfbM den Auftrag ganz zu entziehen. Auch wenn das Wegfallen dieses Auftrages die Existenz der Werkstatt nicht grundsätzlich gefährdet (dieser Auftrag macht etwa 35\% des Betriebsumsatzes aus), so sind damit doch erhebliche Reorganisationen im Betrieb verbunden.

Der Geschäftsführer drängt die Fachanleiter, schnell jemand Anderen für die Bedienung der Maschine fit machen. Diese beschweren sich beim Geschäftsführer über die mangelnde Unterstützung durch die Sozialarbeiterin. Da es in der Werkstatt keinen geeigneten Ersatz für A. gibt soll H. dafür sorgen, dass A. seine Arbeit so bald als möglich wieder aufnimmt. Sie soll sich deswegen auch um die Beilegung der Spannungen im familiären Umfeld von A. kümmern. H. versucht nun, den Druck auf A. zu minimieren. Da sie ihr gutes Vertrauensverhältnis und bisheriges Arbeitsbündnis zu A. nicht gefährden möchte, verschweigt sie A. die ökonomisch und sozial angespannte Situation in der Werkstatt. Gegenüber dem Geschäftsführer spielt sie die angespannte Situation in der Familie herunter und verschweigt, dass der Hausarzt von einer langfristig anhaltenden Verschlechterung des Gesundheitszustandes von A. ausgeht und seine Rückkehr in die Werkstatt sehr kritisch sieht. Da sich die Lage nicht bessert, bekommt H. auch noch Ärger mit C., die mit den Eltern von A. befreundet ist und sich als Gemeindemitglied ehrenamtlich um A. und seine Familie kümmert. C. beschwert sich bei H. darüber, dass die Werkstatt zu viel Druck auf ihren Schützling ausübt, wodurch seine Krankheitssymptome und Verhaltensauffälligkeiten nur verstärkt würden.

An diesem Fall zeigen sich die unterschiedlichen Handlungsmotive, Bezugswerte und Abhängigkeiten der Helfenden einerseits und der wirtschaftlich Verantwortlichen andererseits. A. erhält unentgeltlich und aus christlich-altruistischen Motiven Hilfe von C., ohne das C. für ihre Nächstenliebe eine direkte Gegenleistung, außer vielleicht ein wenig Anerkennung und Dankbarkeit, erwartet. Sie verhält sich solidarisch gegenüber ihrem Gemeindebruder und setzt dafür ihre emotionale und moralische Macht gegenüber den kommunikativen und ökonomischen Machtquellen der Professionellen in der Werkstatt ein. Sollten Anerkennung und Dank von A. gegenüber C. ausbleiben und dieses Reziprozitätsprinzip nicht einhalten, könnte sich $\mathrm{C}$. ohne weitere Begründung und formale Verpflichtung aus dieser informellen Hilfebeziehung zurückziehen. Während die Werkstatt Einkommenseinbußen durch den Produktionsausfall erleidet, ist der Lebensunterhalt von A. durch die Sozialtransfers gesichert. Sein Platz in der Werkstatt ist nur dann gefährdet, wenn er die Beschäftigungskriteri- en und Mitwirkungspflichten der Sozialgesetzgebung nicht mehr erfüllt. Zwischen Geschäftsführung, Sozialdienst und Fachanleitern nehmen die Spannungen und gegenseitige Schuldzuweisungen zu, worunter nun zunehmend auch andere Klienten in der Werkstatt leiden. Es zeigt sich, dass sich die Beteiligten zwischen werterationalen und moralischen Prinzipien auf der einen Seite und zweckrationalen Überlegungen auf der anderen Seite bewegen.

\section{Wohlfahrtsmix und seine Wirkungen auf die die Sorgearbeitenden ihre Hilfebeziehungen und Bewältigungsstrategien von Ungewissheit und Unsicherheit}

Einem über temporäre Allparteilichkeit hinausgehenden, grundsätzlich einseitig-parteilichen und rein anwaltlichen Professionsverständnis sind in diesem Handlungsfeld deutliche Grenzen gesetzt. Die Widersprüchlichkeit von Organisation und Organisationen lässt sich nicht durch einen voluntaristischen Akt auflösen (Eidenschink und Merkes 2021, S. $11 \mathrm{ff}$.). Vereinseitigungen sind mit erheblichen Ambivalenzen und Konflikten verbunden. Das ergibt sich allein schon aus der Tatsache, dass alle Angebote der Sozialwirtschaft überwiegend aus Steuermitteln und Sozialversicherungsbeiträgen finanziert werden. Mitgliedsbeiträge, Spenden und Markterlöse (Warenverkauf, Eintrittsgelder und Teilnahmegebühren etc.) spielen in den meisten Organisationen der Sozialwirtschaft (anders als im Fallbeispiel) eine marginale Rolle. Der sozialrechtliche Rahmen und das Vorhandensein von gesellschaftlich als relevant anerkannter sozialer Probleme sind die zentrale Bedingung für die Inanspruchnahme öffentlicher Mittel (Kohlhoff 2017) (siehe Abb. 2).

In den letzten Jahrzehnten hat sich die Zahl intermediärer Organisationen stark ausgeweitet. $\mathrm{Zu}$ diesen Organisationen gehören die traditionellen Wohlfahrtsverbände ebenso wie kleine gemeinnütze Vereine. In allen diesen Organisationen wirkt sich der Wohlfahrtsmix in besonderer Weise auf den Charakter der Arbeits- und Hilfebeziehungen aus. Sozialstaatliche Instanzen treten gegenüber ihren Klienten (Schutzbefohlenen) in asymmetrischen Beziehungen auf. Die Arbeits- und Hilfebeziehungen in Gemeinschaft und Markt erscheinen zwar symmetrischer, sind aber doch auch durch größere emotionale oder ökonomische Abhängigkeiten geprägt. Auch wenn die dominanten Beziehungsformen in einer hybriden Organisation vermischt werden, bleiben die unterschiedlichen und sich teilweise ausschlieBenden Regulationsprinzipien im Grundsatz erhalten und wirken verdeckt weiter.

Heiner versteht diese Widersprüche nicht als antagonistische Dualismen, sondern als wechselseitig aufeinander 
Abb. 2 Charakter der Hilfebeziehungen in den unterschiedlichen Bereichen der Sozialwirtschaft. (C) Herbert Effinger)

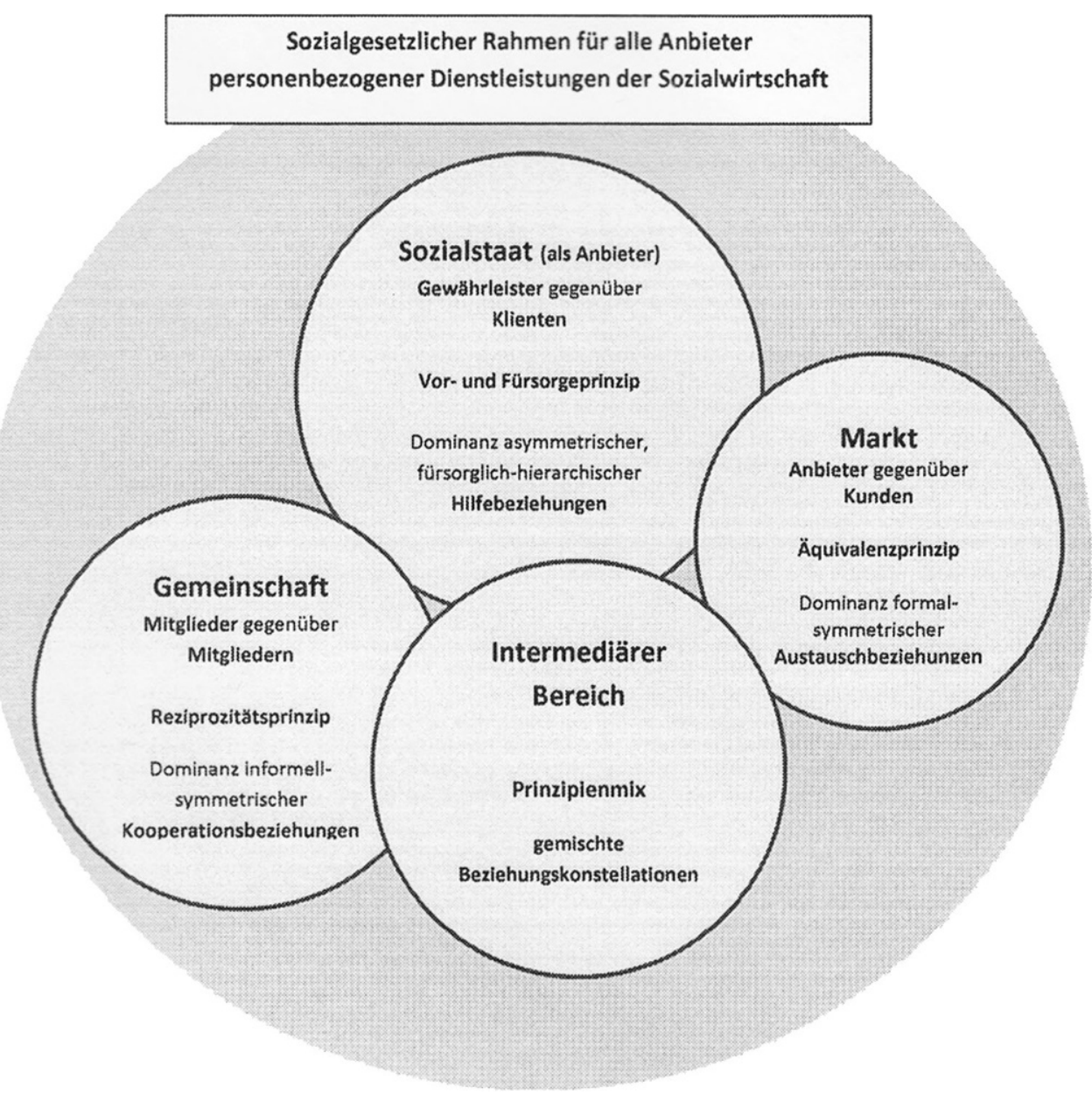

Abb. 3 Konfiguration des Kräftefeldes sozialstaatlich induzierter sozialer personenbezogener Dienstleistungen zur Daseinsvor- und -fürsorge. (C Herbert Effinger (Effinger 2021a, S. 133))

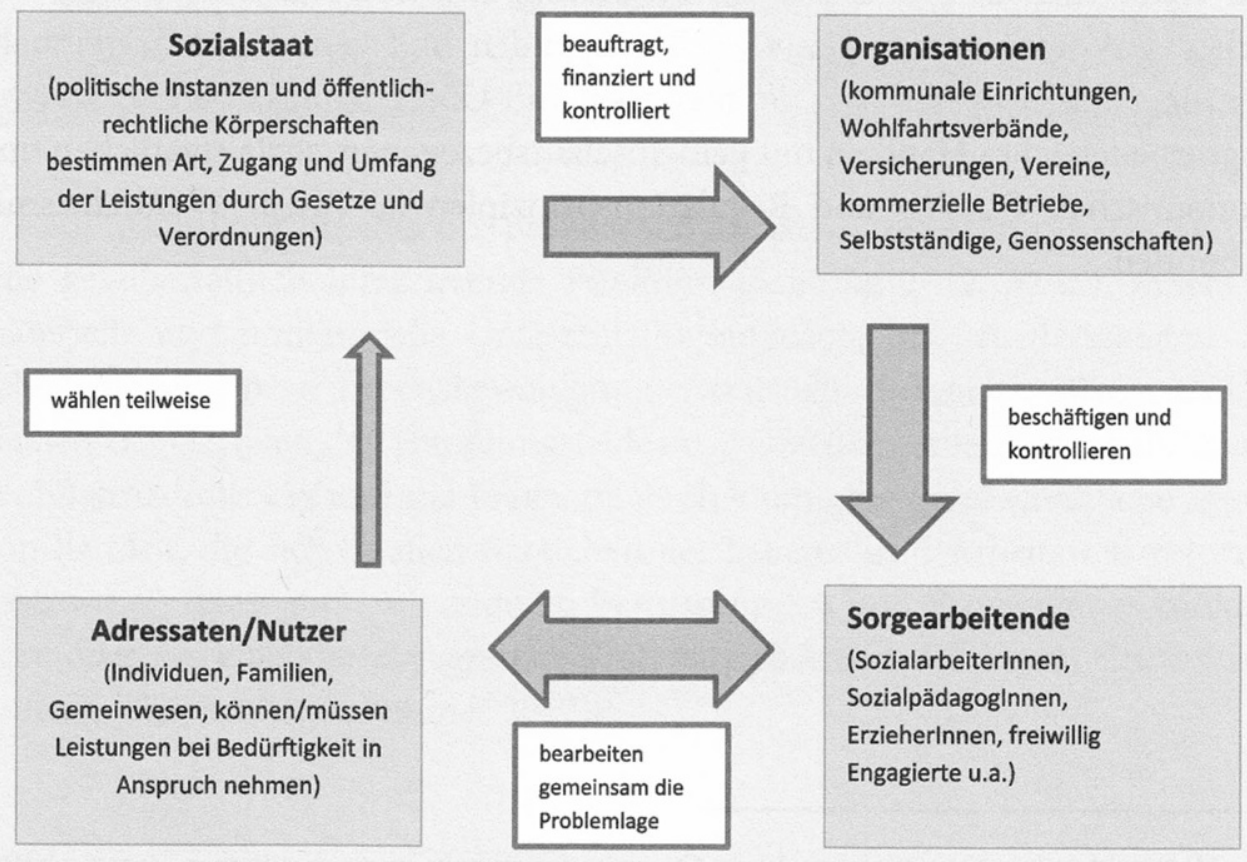


Tab. 1 Widersprüchliche Hilfebedingungen und Aufträge in der Sozialwirtschaft. (@ Herbert Effinger)

\begin{tabular}{|c|c|c|c|c|c|}
\hline Bereich & $\begin{array}{l}\text { Vorbedingung für } \\
\text { ein Hilfsangebot an } \\
\text { „Bedürftige“ }\end{array}$ & Auftrag an die Sorgearbeitenden & $\begin{array}{l}\text { (Heimlicher) Auftrag an Nut- } \\
\text { zer und erwartete Gegenleis- } \\
\text { tung }\end{array}$ & $\begin{array}{l}\text { Primäres } \\
\text { Interventi- } \\
\text { onsmedium }\end{array}$ & $\begin{array}{l}\text { Nutzer- } \\
\text { status }\end{array}$ \\
\hline Gemeinschaft & $\begin{array}{l}\text { Wir helfen Dir, wenn } \\
\text { Du Teil unserer Ge- } \\
\text { meinschaft wirst }\end{array}$ & $\begin{array}{l}\text { Integriere Dich in unser Wertesys- } \\
\text { tem }\end{array}$ & $\begin{array}{l}\text { Assimiliere Dich und sei } \\
\text { konform }\end{array}$ & $\begin{array}{l}\text { Vertrauen } \\
\text { und Solidari- } \\
\text { tät }\end{array}$ & Mitgliec \\
\hline Sozialstaat & $\begin{array}{l}\text { Wir helfen Dir, wenn } \\
\text { Du einen Rechtsan- } \\
\text { spruch auf Hilfe hast }\end{array}$ & $\begin{array}{l}\text { Passe Dich an und begrenze den } \\
\text { Aufenthalt der Klienten in unserem } \\
\text { Bedarfs- und Zuständigkeitssystem }\end{array}$ & $\begin{array}{l}\text { Halte Dich an Deine Pflichten } \\
\text { und Regeln, sei ordentlich }\end{array}$ & $\begin{array}{l}\text { Gesetze } \\
\text { (Rechte und } \\
\text { Pflichten) }\end{array}$ & Klient \\
\hline Markt & $\begin{array}{l}\text { Wir helfen Dir, wenn } \\
\text { Du uns bezahlen } \\
\text { kannst }\end{array}$ & $\begin{array}{l}\text { Verhalte Dich adäquat in unserem } \\
\text { Verwertungssystem }\end{array}$ & $\begin{array}{l}\text { Mach uns nicht zu viel Arbeit } \\
\text { und koste uns nicht mehr als } \\
\text { wir erstattet bekommen }\end{array}$ & Geld & Kunde \\
\hline
\end{tabular}

bezogene Polaritäten (Heiner 2007, S. 46). Sie hebt dabei die Gleichzeitigkeit von Hilfe und Kontrolle, Offenheit und Strukturiertheit der Interventionen, Konsensorientierung und Konfrontation und von Defizit- und Ressourcenorientierung sowie die Asymmetrie in der professionellen Hilfebeziehung als für Sozialwirtschaft konstituierende Elemente hervor. Je nach konkreter Situation und Zuständigkeit (siehe Abb. 3) ergeben sich für sie aus den jeweils unterschiedlichen Kräftekonstellationen besondere „berufliche Anforderungen und professionelle Bewältigungsmuster" (Heiner 2007, S. 429 ff.).

Da die widersprüchlichen Bezugspunkte auch mit gegensätzlichen Verhaltensaufforderungen verbunden sind (siehe Tab. 1), birgt dieses Ausbalancieren im Wohlfahrtsmix ein erhebliches Verunsicherungspotential auf der Entscheidungs- und Handlungsebene und kann letztlich zu ungeeigneten Bewältigungsstrategien und zu erheblichen Teamund Leitungskonflikten beitragen.

\subsection{Wohlfahrtsmix begünstigt Vermeidungsverhalten und organisiertes Misstrauen}

Die mit dem Wohlfahrtsmix einhergehenden Bedrohungsfaktoren können bei den Beteiligten „Vermeidungsziele“ (Kuhl 2010, S. $344 \mathrm{ff}$.) begründen und ein defensives
Vermeidungsverhalten (Effinger 2018, S. 58ff., 2021a, S. $169 \mathrm{ff}$.) befördern. Jede Person, aber auch jede Organisation verfügt zumeist über relativ stabile Vermeidungsmuster bzw. Vermeidungskulturen. Oft erscheinen die Sorgearbeitenden dann allerdings als konfliktscheu, angepasst, wenig kompetent (Reichmann 2018; Scheidgen 2019) oder als kompromisslos. Lernerfahrungen und damit Kompetenzund Organisationsentwicklung werden so behindert.

Aus Team- und Leitungskonflikten können sich je nach Einbindung und Positionsmacht in der Organisation oder der Ausrichtung der Handelnden an einem präferierten Regulationsprinzip unterschiedliche Bedrohungspotentiale ergeben, die unterschiedliche Formen eines defensiven Vermeidungsverhaltens begünstigen (siehe Tab. 2). Darunter verstehe ich verständliche aber ungeeignete Formen der Reduktion von Komplexität, die aus Vereinseitigung und Simplifizierung besteht und den Kern einer komplexen Handlungsherausforderung in einer komplexen Situation ausblendet. Sie kann sich mal mehr in Form der Anpassung oder als ein rebellisch-trotziges Verhaltensmuster zeigen. Während sich die anpassenden Strategien vorwiegend in Form verdeckter Handlungen zeigen, stützen sich die rebellischen Strategien meist auf politisch oder moralisch konnotierte Positionen. Beide Strategien sind oft mit unproduktiven Kommunikationsschleifen, Fehlsteuerungen und kräftezehrenden Konflikten verbunden.

Tab. 2 Bedrohungspotentiale und defensives Vermeidungsverhalten der Sorgearbeitenden in der Sozialwirtschaft. (@ Herbert Effinger)

\begin{tabular}{ll}
\hline Bereich & Unerwünschtes Handeln und Bedrohungspotential \\
\hline Gemeinschaft & $\begin{array}{l}\text { Unsolidarisches Handeln, Ausschluss aus der Ge- } \\
\text { meinschaft, Fraktionierungen und Parteienbildung }\end{array}$ \\
Sozialstaat & $\begin{array}{l}\text { Verletzung von Vorschriften und Überschreitung von } \\
\text { Zuständigkeiten, disziplinarische oder strafbewährte } \\
\text { Konsequenzen }\end{array}$
\end{tabular}

Markt

„Unwirtschaftliches“ Verhalten, überschreiten vorhandener Budgets, Reduktion der entlohnten Arbeitszeit bei nachlassender Nachfrage, betriebsbedingte Kündigung

Mögliches defensives Vermeidungsverhalten

(Schein)Anpassung (so tun als ob-Strategien), Transparenzvermeidung und verdeckte Interventionen, Heimlichkeiten, Konflikten ausweichen und Verzicht auf (wertschätzende) Konfrontation oder persönliche Machtkämpfe, Harmonie um (fast) jeden Preis

Keine, nur informelle oder nicht tatsachengerechte Dokumentation, Dienst nach Vorschrift und Weiterreichen ,heikler“ schwieriger Fälle (Verschiebebahnhof), Datenschutz als Vorwand für „Nichtstun,“ einseitige Schuldzuweisungen

Neue Bedarfe (Diagnosen) erfinden, Kunden länger in einer Maßnahme halten als erforderlich 


\subsection{Wohlfahrtsmix erschwert Vertrauensbildung, Arbeitsbündnisse und Kooperationsbeziehungen}

Ein wechselseitiges Mindestmaß an Vertrauen und vor allem Zutrauen in die Kompetenz eines oder einer Sorgearbeitenden sind nicht nur zentrale Voraussetzungen für ein funktionierendes Arbeitsbündnis und eine gelingende Problembewältigung, sondern auch für das Gelingen der Kooperationsbeziehungen innerhalb einer Organisation und zwischen den beteiligten Hilfesystemen (Wagenblass 2018). ${ }^{9}$

So stehen Sorgearbeitende und ihre Adressaten und Adressatinnen vor der Schwierigkeit, wechselseitig eine vertrauensbasierte, positive Grundhaltung einzunehmen und dem Gegenüber einen Vertrauensbonus zu gewähren, auch dann, wenn aufgrund ihrer bisherigen Erfahrung das Handeln eher von Misstrauen geprägt ist. Erscheint aber einem oder einer der Beteiligten das entgegengebrachte Vertrauen mit Zweifeln besetzt, ergibt sich leicht eine Abwärtsspirale gegenseitigen Misstrauens. Das kann beispielsweise der Fall sein, wenn gegenüber den Adressaten oder Adressatinnen verschwiegen wird, dass eine Maßnahme mit Sanktionsdrohungen verbunden ist und der Eindruck entsteht, dass nicht mit offenen Karten gespielt wird. So bergen die explizit oder implizit ausgesprochenen oder zugeschriebenen, widersprüchlichen Botschaften in diesem komplexen und teilweise undurchschaubaren Funktionssystem ein erhebliches Potenzial für Irritationen, Verunsicherung, Ambivalenzen und soziale Konflikte.

Wenn Entscheidungen (auch die eigenen) in komplexen, unübersichtlichen und widersprüchlichen Situationen zu nicht intendierten Wirkungen geführt haben, neigen viele Sorgearbeitende dazu, egal auf welcher Ebene und in welcher Funktion, die daraus entstandenen Konflikte und Spannungen zu personifizieren und die Schuld dafür jemanden zuzuschreiben, den oder die man entweder fachlich oder moralisch für ungeeignet hält. Misstrauen kennzeichnet so das Organisationsklima.

Je weiter der Arbeitgeber von diesem Arbeitsbündnis räumlich und strukturell entfernt ist, desto eher versuchen Sorgearbeitende, gegenüber ihren Adressaten und Adressaten Vertrauen aufzubauen und sich schützend vor ihr Klientel zu stellen und damit Anforderungen aus der Organisation von ihnen fernzuhalten. Gleichzeitig verstärken sie aber das Misstrauen von Kollegen und Kolleginnen oder der Leitung. Die Nähe zu den Einen fördert die Distanz zu den Anderen. So werden die nicht am unmittelbaren Hilfeprozess beteiligten Träger und zuständigen Ämter (Kostenträger und Aufsichtsbehörden) nicht als Teil des Hilfesystems und Kooperationspartner bei der Problembearbeitung

\footnotetext{
${ }^{9}$ In Disziplin und Profession wird diese Problematik auch unter den Begriffen von professioneller Nähe-Distanz diskutiert (Dörr 2019).
}

betrachtet, sondern als einschränkende Instanzen, denen gegenüber man sich möglichst bedeckt verhält.

Scharmer und Käufer (2017) beschreiben solche Phänomene als Folge von Entkopplungsprozessen in arbeitsteiligen, modernen Gesellschaften. Wenn sich Sorgearbeitende selbst primär im Kontext von Gemeinschaft verorten, von ihren Adressaten und Adressatinnen aber als mit Kontrollmächten ausgestattete Repräsentanten sozialpolitischer Institutionen gesehen werden, werden aus Mitgliedern oder Freunden „Verkäufer“ von Angeboten und Personen, denen man nur begrenzt trauen kann.

\subsection{Wohlfahrtsmix begünstigt Stress}

Effizienz und Effektivität sind an Ziele gebunden, an denen man den Erfolg oder Misserfolg einer Intervention messen können sollte. Wenn aber Ziele verfolgt werden sollen, die einem von anderen Personen oder Institutionen abverlangt werden, nicht von den eigenen Gefühlen und von eigener Motivation getragen werden, entsteht Stress (Martens und Kuhl 2020, S. 32). Solche Situationen sind in der Sozialwirtschaft angesichts der unterschiedlichen Interessenlagen durchaus typisch. Insgesamt ist das Handlungsfeld der Sozialen Arbeit von einem emotionalen Paradox gekennzeichnet. Anstelle von besonders verlässlichen Formen des Vertrauens und Zutrauens finden wir oft psychisch besonders belastende Strukturen und Prozessdynamiken, die eher Misstrauen begünstigen. Das scheint ein Grund dafür zu sein, dass die Beschäftigten in diesem Bereich besonders häufig psychische Erkrankungen aufweisen, überproportional häufig Mobbing vorkommt und eine hohe Personalfluktuation zu verzeichnen ist (DAK 2020, S. $61 \mathrm{ff}$.).

\section{Fazit}

Aus der Perspektive eines Sozialarbeiters, Supervisors und Organisationsberaters ist es oftmals schwer erträglich, zu sehen, wie viele Energien Mitarbeitende und Leitende bei der Bewältigung von Konflikten und unlösbaren Aufgaben verschwenden, die sich aus normativer Überfrachtung und mangelnder Berücksichtigung der komplexen Systemdynamiken ergeben. Soziale Systeme können nicht ohne Konflikte auskommen. Es gibt keine vollkommen konsistenten und widerspruchsfreien Zielkonzepte. Nach meiner Erfahrung sind Sozialarbeitende leider recht oft nicht besonders an Organisationsfragen und der Mitgestaltung ihrer Organisation interessiert. Obwohl ein Großteil der Arbeit darin besteht, Unterstützung in einem stark versäulten Hilfesystem zu organisieren und zu koordinieren, wird Organisation von vielen Sorgearbeitenden als ein lästiges Element empfunden, das sie mit unangemessenen Anforderungen ihrer Leitungen nur von der „eigentlichen“ Arbeit mit ih- 
ren Adressaten und Adressatinnen abhält. Sozialarbeitende müssen zwar viel organisieren, sind aber oft selbst schlecht organisiert.

Es ist ,ein großer Unterschied, ob Sozialarbeiter eine für professionelle Arbeitsweisen geeignete administrative Struktur nur fordern, (,von denen da oben“), oder ob sie die Herstellung und ständige Überprüfung der Funktionstüchtigkeit dieser Struktur für die Arbeit mit Klienten zu ihrer ureigenen Aufgabe machen (Müller 2012, S. 63).“ Wenn aber Organisationsentwicklung nicht als Teil ihres professionellen Auftrages, sondern eher als einen Störfaktor betrachtet wird, stellt sich die Frage, inwieweit die Organisationen und deren Leitungen sich dieser Frage so annehmen, dass Sozialarbeitende Organisation als Ressource wahrnehmen, an deren Gestaltung sie gern partizipieren.

Will man nicht einem naiven Glauben folgen, man könne in einem hochkomplexen, kapitalistischen Gesellschaftssystem markt- und betriebswirtschaftliche Elemente oder sozialstaatliche Kontrolle aus dem Subsystem Sozialwirtschaft grundsätzlich ausklammern, dann müssen Sorgearbeitende und Organisationen lernen, zu akzeptieren, dass die Rationalität von Organisation, die sich aus einer Mischung gemeinschaftlicher, sozialstaatlicher und ökonomischer Regulierung ergibt, nicht konfliktfrei mit der Rationalität und Logik professioneller und solidarischer Hilfebeziehungen vereinbar ist.

Auch Organisationen der Sozialwirtschaft müssen nach wirtschaftlichen Kriterien arbeiten. Um ihre Angebote und ihren Aufwand dafür gegenüber der Öffentlichkeit zu legitimieren, sollten sie in der Lage sein, nach den ihnen eigenen, professionellen Kriterien die Effizienz und Effektivität diese Aufwände zu begründen. Und so spricht auch nichts dagegen, wenn sie marktwirtschaftliche und betriebswirtschaftliche Instrumente für die Existenzsicherung und Angebotsverbesserung nutzen, wenn diese nicht nur der betrieblichen und sozialen Ressourceneinsparung, sondern auch einer angemessenen Bedarfsdeckung dienen. Nach gut 30 Jahren „Neuer Steuerung“ zeigt sich aus der Perspektive eines „Marktbeobachters“ jedoch, dass es sich bei diesen Instrumenten oftmals um nicht mehr als um reine Add Ons handelt, die kaum mit den Logiken und den Anforderungen an Soziale Arbeit verbunden sind

Mein Eindruck ist, primär wertrationale Strategien der Disziplin und Profession Sozialer Arbeit auf der einen und primär zweckrationale Strategien von Politik, Wirtschaft und Management auf der anderen Seite stehen sich heute unversöhnlicher gegenüber als das noch vor 30 Jahren der Fall war. Ihre Strategien erscheinen in ihrer Polarisierung als komplementär und wirken mit ihren jeweiligen Feindbildern mit Begriffen wie „Managerealisierung“ auf der einen und „Moralisierung “ auf der anderen Seite selbsterhaltend bzw. selbstverstärkend (Polutta 2014). Dass das Verhältnis von Arbeitsbündnis und Organisation so wenig im Fokus der Sozialarbeitenden steht, hat auch damit zu tun, dass sich im Studium der Sozialen Arbeit der Fokus stark auf die Frage richtet, wie Sicherheit und Vertrauen entstehen kann. So richtig und wichtig das ist, so ist das m.E. nur dann erfolgversprechend, wenn man auch versteht, wie in unsicheren Kontexten Misstrauen entsteht, wie sich dieses minimieren und nutzen lässt und wie auch unter Misstrauen und Unsicherheit gelingende Unterstützungsprozesse möglich werden können (Effinger 2021b).

Sozialarbeitende sollten Experten bzw. Expertinnen für ein situationsorientiertes Handeln unter den Bedingungen von Ungewissheit und Unsicherheit sein. Der beschriebene Wohlfahrtsmix bietet nicht nur Nachteile, sondern auch Vorteile. Bewertet man Vermeidungsverhalten der Sozialarbeitenden und ihres Managements nicht als maladaptiv oder inkompetent, sondern als ein adaptives Verhalten in hybriden und wenig durchschaubaren Organisationsstrukturen und Organisationsdynamiken, stellen sich besondere Anforderungen an die Forschung, Theorie und Praxis, Aus-, Fort- und Weiterbildung und das Management, die darin liegen, pragmatische Strategien und wirkungsvolle Konzepte für einen bedarfsgerechten Ausgleich der unterschiedlichen und gegensätzlichen Regulationsprinzipien zu entwickeln.

Funding Open Access funding enabled and organized by Projekt DEAL.

Open Access Dieser Artikel wird unter der Creative Commons Namensnennung 4.0 International Lizenz veröffentlicht, welche die Nutzung, Vervielfältigung, Bearbeitung, Verbreitung und Wiedergabe in jeglichem Medium und Format erlaubt, sofern Sie den/die ursprünglichen Autor(en) und die Quelle ordnungsgemäß nennen, einen Link zur Creative Commons Lizenz beifügen und angeben, ob Änderungen vorgenommen wurden.

Die in diesem Artikel enthaltenen Bilder und sonstiges Drittmaterial unterliegen ebenfalls der genannten Creative Commons Lizenz, sofern sich aus der Abbildungslegende nichts anderes ergibt. Sofern das betreffende Material nicht unter der genannten Creative Commons Lizenz steht und die betreffende Handlung nicht nach gesetzlichen Vorschriften erlaubt ist, ist für die oben aufgeführten Weiterverwendungen des Materials die Einwilligung des jeweiligen Rechteinhabers einzuholen.

Weitere Details zur Lizenz entnehmen Sie bitte der Lizenzinformation auf http://creativecommons.org/licenses/by/4.0/deed.de.

\section{Literatur}

Aldred, J. (2020). Der korrumpierte Mensch. Die ethischen Folgen wirtschaftlichen Denkens. Klett-Cotta: Stuttgart. 2. Aufl. 2021

Apelt, M., \& Senge, K. (Hrsg.). (2015). Organisation und Unsicherheit. Wiesbaden: Springer.

BAG WfbM (2021). Bundesarbeitsgemeinschaft Werstätten für behinderte Menschen. Die BAG WfbM 17.6.21, gesehen 1.9.2021 https://www.bagwfbm.de/page/24. Zugegriffen: 1. Sep. 2021.

Bode, I., Evers, A., \& Klein, A. (Hrsg.). (2008). Bürgergesellschaft als Projekt: Eine Bestandsaufnahme zu Entwicklung und Förderung zivilgesellschaftlicher Potenziale in Deutschland. Eine Bestandsaufnahme zur Entwicklung und Förderung zivilgesellschaftlicher Potenziale in Deutschland. Wiesbaden: VS. 
Böhle, F. (2017). Subjektivierendes Handeln - Anstöße und Grundlagen. In F. Böhle (Hrsg.), Arbeit als Subjektivierendes Handeln. Handlungsfähigkeit bei Unwägbarkeiten und Ungewissheit (S. 3-42). Wiesbaden: Springer.

Brinkmann, V. (Hrsg.). (2014). Sozialunternehmertum. Baltmannsweiler: Schneider Verlag.

DAK (2020). Gesundheitsreport 2020. Stress in der modernen Arbeitswelt. https://www.dak.de/dak/bundesthemen/gesundheitsreport2020-2371690.html\#/. Zugegriffen: 22. Mai 2021.

Dörr, M. (Hrsg.). (2019). Nähe und Distanz. Ein Spannungsfeld pädagogischer Professionalität (4. Aufl.). Weinheim, Basel: Beltz Juventa.

Effinger, H. (1990). Individualisierung und neue Formen der Kooperation. Bedingungen und Wandel alternativer Arbeits- und Angebotsformen. Wiesbaden: Deutscher Universitätsverlag.

Effinger, H. (1993). Soziale Dienste zwischen Gemeinschaft, Markt und Staat. In H. Effinger \& D. Luthe (Hrsg.), Sozialmärkte und Management (Bd. 26, S. 13-39). Bremen: Universität Bremen. Forschungsprojekt Arbeit und Bildung.

Effinger, H. (2018). Beratung in der Sozialwirtschaft. Ungewissheiten als Chance kreativer Problemlösungsstrategien. Göttingen: Vandenhoeck \& Ruprecht.

Effinger, H. (2021a). Soziale Arbeit im Ungewissen. Mit Selbstkompetenz aus Eindeutigkeitsfallen. Weinheim Basel: Beltz Juventa.

Effinger, H. (2021b). Ungewissheitsbewältigung und akademische Professionalität. Herausforderungen für das Studium Sozialer Arbeit. Soziale Arbeit, 5, 162-169.

Effinger, H., \& Körber, K. (1994). Sozialunternehmer, Freiberufler oder Bedienstete? Professionalisierung im Intermediären Bereich. neue praxis, 1(94), 46-56.

Eidenschink, K., \& Merkes, U. (2021). Entscheidungen ohne Grund. Organisationen verstehen und beraten. Eine Metatheorie der Veränderung. Göttingen: Vandenhoeck \& Ruprecht.

Evers, A., \& Ewert, B. (2010). Hybride Organisationen im Bereich sozialer Dienste. Ein Konzept, sein Hintergrund und seine Implikationen. In Th Klatetzki (Hrsg.), Soziale personenbezogene Dienstleistungsorganisationen. Soziologische Perspektiven (S. 103-128). Wiesbaden: VS.

Evers, A., Heinze, R. G., \& Olk, Th (Hrsg.). (2011). Handbuch Soziale Dienste. Wiesbaden: Springer.

Gahleitner, S.B. (2017). Soziale Arbeit als Beziehungsprofession: Bindung, Beziehung und Einbettung professionell ermöglichen. Weinheim Basel: Beltz Juventa.

Gesmann, St , \& Merchel, J. (2019). Systemisches Management in Organisationen der Sozialen Arbeit. Handbuch für Studium und Praxis. Heidelberg: Carl-Auer-Systeme Verlag.

Grunwald, K., \& Langer, A. (Hrsg.). (2018). Sozialwirtschaft. Handbuch für Wissenschaft und Praxis. Baden-Baden: Nomos.

Hackenberg, H., \& Empter, St (Hrsg.). (2011). Social Entrepreneurship - Social Business. Für die Gesellschaft unternehmen. Wiesbaden: VS.

Harrison, R., \& Lomi, A. (Hrsg.). (2015). Garbage can model of organizational choice: lookin forward at forty. Bingley: Emerald Publishing Ltd.

Heiner, M. (2007). Soziale Arbeit als Beruf. Fälle - Felder - Fähigkeiten. München: Reinhardt.

Heiner, M. (2010). Kompetent handeln in der Sozialen Arbeit. München: Reinhardt. 3. Aufl. 2018

Heinze, R. G. (2011). Soziale Dienste und Beschäftigung. In Evers, et al. (Hrsg.), Handbuch Soziale Dienste (S. 168-186). Wiesbaden: Springer.

Kohlhoff, L. (2017). Finanzierung der Sozialwirtschaft. Eine Einführung (2. Aufl.). Wiesbaden: Springer.

Kolhoff, L., \& Grunwald, K. (Hrsg.). (2018). Aktuelle Diskurse in der Sozialwirtschaft I. Wiesbaden: Springer VS.
Kuhl, J. (2010). Lehrbuch der Persönlichkeitspsychologie. Motivation, Emotion und Selbststeuerung. Göttingen, Bern, Wien: Hogrefe.

Kurz-Adam, M. (2018). Profession ohne Auftrag? Vom Verschwinden der Hilfe in einem helfenden Beruf. пеие praxis, 5, 496-502.

Martens, J.-U., \& Kuhl, J. (2020). Die Kunst der Selbstmotivierung: Neue Erkenntnisse der Motivationsforschung praktisch nutzen (6. Aufl.). Stuttgart: Kohlhammer.

Mazari, S. (2021). Erwerbsverhältnisse und Intersektionalität in der Sozialen Arbeit. Soziale Arbeit, 5, 184-190.

Müller, B. (2012). Professionell helfen: Was das ist und wie man das lernt. Die Aktualität einer vergessenen Tradition Sozialer Arbeit. Ibbenbühren: Münstermann.

Oechler, M. (2009). Soziale Arbeit als Dienstleistung. In Dienstleistungsqualität in der Sozialen Arbeit. Wiesbaden: VS.

Polutta, A. (2014). Managerealisierung. In I.G.F.H. Düring, H.U. Krause, F. Peters, R. Rätz, N. Rosenbauer \& M. Vollhase (Hrsg.), Kritisches Glossar Hilfen zur Erziehung. Frankfurt a.M.: Eigenverlage. https://igfh.de/publikationen/kritisches-glossar/ managerialisierung. Zugegriffen: 30. August 2021.

Reichmann, U. (2018). Schwierige Fälle - konfliktträchtige Entscheidungen. Fachkräftekonflikte als Tabuthema der Jugendhilfe. Opladen, Farmington: Hills: Budrich.

Scharmer, C. O., \& Käufer, K. (2017). Von der Zukunft her führen. Von der Egosystem- zur Ökosystem-Wirtschaft. Theorie U in der Praxis. Heidelberg: Carl-Auer.

Scheidgen, A. (2019). Entscheiden als Aspekt professioneller Kompetenz angehender Fachkräfte der Sozialen Arbeit. Modellierung und empirische Überprüfung eines Konstrukts. Opladen, Berlin, Toronto: Budrich.

Schneiders, K. (2020). Sozialwirtschaft und Soziale Arbeit. Stuttgart: Kohlhammer.

Schröder, C. (2017). Emotionen und professionelles Handeln in der Sozialen Arbeit. Eine Ethnographie der Emotionsarbeit im Handlungsfeld der Heimerziehung. Wiesbaden: Springer.

Schütze, F. (2021). Professionalität und Professionalisierung in pädagogischen Handlungsfeldern: Soziale Arbeit. Opladen, Toronto: Barbara Budrich.

Wagenblass, S. (2018). Vertrauen. In H. U. Otto, H. Thiersch, R. Treptow \& H. Ziegler (Hrsg.), Handbuch Soziale Arbeit. Grundlagen der Sozialarbeit und Sozialpädagogik (6. Aufl. S. 1803-1814). München: Ernst Reinhardt.

Wendt, W. R. (2017). Sozialwirtschaft. In Fachlexikon der Sozialen Arbeit (8. Aufl. S. 856-857). Berlin: Nomos. Herausgegeben vom Deutschen Verein für öffentliche und private Fürsorge e. V.

Wöhrle, A., Gruna, P., Kolhoff, L., Kortendieck, G., Nöbauer, B., Tabatt-Hirschfeldt, A., \& Zillmann, R. (2019). Personalmanagement - Personalentwicklung. Studienkreis Sozialwirtschaft. Baden-Baden: Nomos.

Zobrist, P., \& Kähler, H.D. (2017). Soziale Arbeit in Zwangskontexten. München: Reinhardt. 
682

H. Effinger

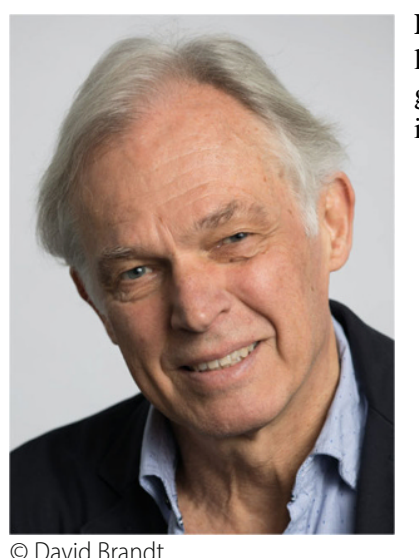

Prof. (em) Dr. Herbert Effinger lehrte Soziale Arbeit an der Evangelischen Hochschule Dresden und ist als Supervisor und Coach tätig

(c) David Brandt

Springer 